\title{
Intersections
}

Canadian Journal of Music

Revue canadienne de musique

\section{Thomas Christensen, dir., The Cambridge History of Western Music Theory. Cambridge: Cambridge University Press, 2002. xxiii, 998 p. ISBN 0-521- 62371-5 (couverture rigide)}

\section{Bruno Gingras}

Volume 25, numéro 1-2, 2005

URI : https://id.erudit.org/iderudit/1013314ar

DOI : https://doi.org/10.7202/1013314ar

Aller au sommaire du numéro

Éditeur(s)

Canadian University Music Society / Société de musique des universités canadiennes

ISSN

1911-0146 (imprimé)

1918-512X (numérique)

Découvrir la revue

Citer ce compte rendu

Gingras, B. (2005). Compte rendu de [Thomas Christensen, dir., The Cambridge History of Western Music Theory. Cambridge: Cambridge University Press, 2002. xxiii, 998 p. ISBN 0-521- 62371-5 (couverture rigide)]. Intersections, 25(1-2), 233-240. https://doi.org/10.7202/1013314ar

Copyright ( C Canadian University Music Society / Société de musique des universités canadiennes, 2005
Ce document est protégé par la loi sur le droit d'auteur. L'utilisation des services d’Érudit (y compris la reproduction) est assujettie à sa politique d'utilisation que vous pouvez consulter en ligne.

https://apropos.erudit.org/fr/usagers/politique-dutilisation/ 
ment prochain d'une esthétique de la stabilité : « J'aimerais suggérer que la prochaine période (à moins que nous ne soyons déjà dedans) sera une période de stagnation stylistique, une période caractérisée non par le développement linéaire et cumulatif d'un style fondamental, mais par la coexistence d'une multiplicité de styles passablement différents dans un état de constance fluctuant et dynamique. " (Meyer 1967, 98, cité en p. 1395).

Musiques répond à la nécessité épistémologique de revivifier la recherche musicologique en proposant des pistes de lecture inédites, en sortant l'objet musical de lui-même et en l'ouvrant aux autres disciplines des sciences humaines. Le recours à des perspectives diverses issues de disciplines différentes permettra certes aux musicologues traditionnels, formés dans les écoles de musique, de comprendre que l'on peut être spécialiste de la musique sans avoir été formé à l'école de la musicologie.

\section{RÉFÉRENCES}

Gervasoni, Pierre. 2003. "Sons discordants sur la musique du XXe siècle ». Le Monde (20 juin) : IX.

Meyer, Leonard B. 1967. Music, the Arts, and Ideas: Patterns and Predictions in Twentieth-Century Culture. Chicago : University of Chicago Press.

Nattiez, Jean-Jacques. 1968. Fidel Castro. Coll. " Destins politiques ». Paris : Seghers.

- 1999. Proust musicien, $2^{\mathrm{e}}$ éd. Coll. "Musiques ". Paris : Christian Bourgois.

Shepherd, John, David Horn et al. 2003-. Continuum Encyclopedia of Popular Music of the World. New York: Continuum.

Jean Nicolas De Surmont

Thomas Christensen, dir. The Cambridge History of Western Music Theory. Cambridge : Cambridge University Press, 2002. xxiii, 998 p. ISBN 0-52162371-5 (couverture rigide).

Édité par Thomas Christensen, spécialiste de l'histoire de la théorie musicale et auteur entre autres d'une étude remarquable de l'œuvre théorique de Rameau (Rameau and Musical Thought in the Enlightenment, paru en 1993), le Cambridge History of Western Music Theory comble une lacune béante dans le domaine de l'histoire de la théorie musicale. En effet, si l'on fait exception de quelques efforts louables, peu nombreuses sont les publications récentes qui ont pour ambition de couvrir de façon exhaustive l'histoire de la théorie musicale occidentale. On doit mentionner la monumentale mais inachevée Geschichte der Musiktheorie, encyclopédie en 15 volumes élaborée sous la direction de Frieder Zaminer et Thomas Ertelt (1984- ), citée à plusieurs reprises dans le Cambridge History of Western Music Theory, dont les dimensions imposantes, sans parler de l'utilisation de la langue allemande, pourraient cependant rebuter plus d'un lecteur nord-américain. Parmi quelques 
ouvrages d'envergure plus modeste, signalons l'étude de Carl Dahlhaus (1968) sur les origines de la tonalité, ainsi qu'un survol historique des théories de l'harmonie publié par Matthew Shirlaw (1917), déjà vieux de près d'un siècle. Sinon, il faut remonter au XIX' ${ }^{\mathrm{e}}$ siècle pour trouver des encyclopédies de l'histoire de la théorie musicale dignes de ce nom, sous les plumes respectives d'Hugo Riemann (1898) et de François-Joseph Fétis (1840), dont les travaux souffrent malheureusement des préjugés de leurs auteurs, en plus d'accuser un âge certain.

Le Cambridge History of Western Music Theory s'avère un exemple probant de collaboration académique au plus haut niveau : pas moins de 32 auteurs ont contribué à sa rédaction, chacun d'entre eux s'étant chargé d'un domaine dans lequel il fait figure de sommité. À ce titre, les universités canadiennes sont avantageusement représentées, puisque quatre professeurs y œuvrant ont été invités à collaborer à cet ouvrage, soit William Caplin (théories du rythme aux XVIII ${ }^{e}$ et XIX ${ }^{\mathrm{e}}$ siècles, chap. 21), Henry Klumpenhouwer (théories harmoniques dualistes du XIX ${ }^{\mathrm{e}}$ siècle, chap. 14), Catherine Nolan (théorie musicale et mathématiques, chap. 10), et Peter Schubert (pédagogie du contrepoint durant la Renaissance, chap. 16).

L'ordre de présentation des chapitres, qui a de toute évidence fait l'objet d'une réflexion assidue, est basé sur une suggestion de Dahlhaus. Dans Geschichte der Musiktheorie, ce dernier propose une division de la théorie musicale en trois branches : une tradition spéculative, considérée comme " contemplation ontologique des systèmes tonaux ", une tradition pratique, qui concerne la "régulation et la coordination des systèmes tonaux " appliquée à la composition, et une tradition analytique ou descriptive, qui examine les œuvres musicales en tant qu'entités individuelles, dans le but de comprendre leurs particularités spécifiques (Zaminer et Ertelt, dir. 1984-, vol. 1, 6-9; cité en p. 13-14.) ${ }^{1}$. Cette division tripartite est précédée ici d'une section visant à définir la discipline ainsi que ses enjeux principaux.

La première partie vise donc à délimiter la théorie musicale en tant que domaine de connaissance. Le premier chapitre, rédigé par Leslie Blasius, trace les subdivisions de la discipline et commente l'évolution historique de ces frontières. Robert Wason, dans le chapitre 2, discute de l'évolution des liens entre la pédagogie et la formulation théorique de concepts musicaux. Le chapitre 3, dont l'auteur est Nicholas Cook, constitue une réflexion fascinante sur les fondements épistémologiques de la théorie musicale, examinés à la lumière des théories foucauldiennes.

Bien que la deuxième partie, qui aborde la branche spéculative de la théorie musicale, puisse a priori sembler d'un intérêt limité pour le théoricien qui se soucie principalement de l'analyse de la musique tonale et post-tonale, elle s'avère éminemment instructive pour celui qui désire se familiariser avec les

1 " Ontological contemplation of tone systems "; " regulation and coordination of tone systems ". Les traductions sont de l'auteur de la présente recension. 
perspectives socio-historiques sous-tendant la discipline, et tout particulièrement avec sa dimension empirique, qui constitue peut-être l'une de ses ramifications les plus dynamiques à l'heure actuelle, en raison notamment de l'utilisation croissante de méthodes scientifiques. Le chapitre 4 (Thomas J. Mathiesen) nous propose une introduction à la théorie musicale de l'Antiquité grecque, dont découle, indirectement à tout le moins, l'essentiel de la théorie musicale subséquemment élaborée en Occident. La réappropriation des théories de l'Antiquité grecque dans la théorie musicale médiévale, et leur adaptation aux usages liturgiques associés au christianisme, qui ne s'est pas faite sans de nombreux détournements de sens, est décrite de façon colorée par Calvin Bower dans le chapitre 5. Le chapitre 6 (Jan Herlinger) s'intéresse aux théories médiévales de l'accord (ajustement des hauteurs de notes dans la gamme), et en particulier aux diverses propositions de division du monocorde. L'évolution des théories du tempérament et de l'accord au cours de la Renaissance fait l'objet du chapitre suivant (Rudolf Rasch), qui doit être loué pour sa clarté dans l'exposition d'un sujet parfois rébarbatif pour qui ne possède pas de solides bases mathématiques. Penelope Gouk nous entretient ensuite des liens étroits entre la conception harmonique de l'univers, exprimée sous sa forme la plus connue dans le mythe de l'harmonie des sphères, et la révolution scientifique de la Renaissance (chap. 8), tandis que le chapitre 9, écrit conjointement par Burdette Green et David Butler, montre comment cette révolution scientifique entraînera le développement d'une véritable musicologie expérimentale, ou "Tonpsychologie ". On notera l'arrangement chronologique des chapitres 4 à 9. Finalement, les liens souvent étroits entre la théorie musicale et les mathématiques, présents depuis l'Antiquité, sont explorés dans le chapitre 10, sous la plume de Catherine Nolan.

La troisième partie, intitulée "traditions régulatrices", traite essentiellement de la codification de la pratique musicale sous toutes ses formes, soit la composition en premier lieu, mais aussi l'interprétation, l'improvisation, et les systèmes d'enseignement de la musique. Cette partie se divise en quatre sous-sections. La première discute des théories et des systèmes mis de l'avant, depuis le Haut Moyen Âge jusqu'au XIX ${ }^{e}$ siècle, afin de définir les notions de mode, de gamme, ainsi que de tonalité, et de répertorier systématiquement tout phénomène musical à l'aune de ces catégories. Le chapitre 11, soumis par David Cohen, nous propose un survol des principaux traités musicaux du Haut Moyen Âge, en s'attardant davantage sur l'évolution des conceptions médiévales associées à l'idée de note, de gamme, et de mode. Cet article touffu est parsemé de nombreux tableaux qui contribuent grandement à clarifier l'exposé. Cristle Collins Judd opte, dans le chapitre 12, pour une approche socio-historique du concept de modalité, centrée autour de l'œuvre de Zarlino, au lieu d'un compte-rendu méthodique des théories modales de la Renaissance. Gregory Barnett (chap. 13) discute ensuite de la transition graduelle de la modalité à la tonalité au cours du XVII ${ }^{\mathrm{e}}$ siècle, en mettant en valeur les particularités associées à la culture musicale de divers pays d'Europe 
occidentale. Enfin, le chapitre 14 (Henry Klumpenhouwer), qui traite du dualisme harmonique au XIX ${ }^{e}$ siècle, semble chercher à réhabiliter certains aspects de cette théorie qui ne sont plus tout à fait au goût du jour.

La seconde sous-section présente les théories développées en rapport avec la composition, accordant une place de choix au contrepoint. Sarah Fuller nous offre un vibrant compte-rendu des théories compositionnelles au Moyen Âge (chap. 15), tandis que Peter Schubert s'intéresse, dans le chapitre 16, à la pédagogie du contrepoint à l'époque de la Renaissance. Dans le chapitre 17, Albert Cohen examine les liens entre l'interprétation et la composition, et s'intéresse particulièrement à l'improvisation, élément majeur de la pratique musicale occidentale jusqu'au XVIII ${ }^{e}$ siècle. Le chapitre 18 (Ian Bent) se distingue en ce qu'il est entièrement consacré à un traité, le Gradus ad Parnassum de Fux, et à son influence sur la pédagogie du contrepoint, qui se fait encore sentir de nos jours. La discussion porte davantage sur la forme du traité et sur la façon dont sont exposées les règles, que sur les préceptes euxmêmes avec lesquels nous sommes déjà familiers. John Covach (chap. 19) traite de l'évolution historique des théories concernant la musique dodécaphonique au $\mathrm{XX}^{\mathrm{e}}$ siècle, en insistant sur la dimension essentiellement " prescriptive " de cette théorie, initialement destinée à systématiser le langage musical des compositeurs désireux de s'aventurer dans cette voie.

Les théories du rythme sont abordées dans la troisième sous-section. Le chapitre 20 (Anna Maria Busse Berger) se veut une narration historique de l'évolution de la théorie rythmique du Haut Moyen Âge à la Renaissance, à travers les écrits des principaux auteurs qui se sont penchés sur la question. À l'inverse, William Caplin a préféré regrouper les formulations théoriques autour d'un thème commun, plutôt que sur une base individuelle, dans son exposé des théories rythmiques aux XVIII ${ }^{e}$ et $\mathrm{XIX}^{e}$ siècles, dans lequel il parvient à discourir avec aisance de concepts quelque peu abscons, tels que les théories rythmiques de Hauptmann (chap. 21) ${ }^{2}$. Justin London prend le relais au chapitre 22 , en nous présentant l'éclosion de multiples théories rythmiques au XXe siècle, regroupées en quatre courants principaux (énergétique, schenkérien, hiérarchique et post-tonal). Visiblement, les chapitres composant cette section ont été étroitement coordonnés.

La quatrième sous-section s'intéresse au concept moderne de tonalité, depuis Rameau jusqu'à Schenker. C'est d'ailleurs la seule section qui met véritablement l'emphase sur l'œuvre de théoriciens individuels (Rameau et Schenker), alors que l'ouvrage semble avoir privilégié en général les regroupements par thèmes et sujets apparentés, plutôt qu'autour de personnalités marquantes de la théorie musicale. Le chapitre 23 (Bryan Hyer) se veut un essai provoquant sur l'usage et les multiples définitions du terme " tonalité ",

2 Caplin parle d'ailleurs de « théories qui pourraient paraitre quelque peu étranges, sinon carrément bizarres aux yeux du lecteur contemporain " ( " formulations that might strike the modern reader as somewhat strange, if not downright bizarre ", p. 679). 
et sur ses ramifications culturelles et idéologiques, doublé d'une réflexion sur la pertinence de l'orientation progressiste qui semble inéluctablement teinter le discours historique sur la discipline. Le chapitre 24, soumis par Joel Lester, est un compte-rendu lucide des théories de Rameau et de leur impact ultérieur sur la théorie musicale. David Bernstein nous présente, dans le chapitre 25, un remarquable exposé des principales théories harmoniques ayant prospéré dans l'orbite austro-prussienne au XIX ${ }^{e}$ siècle. Dans le chapitre 26, William Drabkin réussit le tour de force de résumer les principaux éléments de la théorie schenkérienne en une trentaine de pages.

La quatrième et dernière partie, "traditions descriptives ", est consacrée aux méthodes d'analyse musicale qui visent à rendre compte d'une œuvre individuelle. Le chapitre 27 (Patrick McCreless) propose un résumé des travaux des principaux théoriciens ayant fait usage de concepts issus de la rhétorique. Scott Burnham (chap. 28) se charge des questions liées à la forme, et notamment à la forme sonate, en mettant l'accent sur les implications analytiques découlant d'une approche historique qui prend en compte les notions de convention stylistique et historique. L'analyse thématique et motivique est présentée dans le chapitre 29 par Jonathan Dunsby, qui survole brièvement trois approches distinctes : la technique de variation évolutive ("developing variation ») de Schoenberg, l'approche sémiotique, et l'analyse des ensembles de catégories de hauteur. Les idées d'un groupe de théoriciens germaniques tels que Kurth et Schering, caractérisées par une vision dynamique de la musique mettant l'accent sur la notion de mouvement, sont exposées au chapitre 30, une contribution de Lee Rothfarb. Le chapitre 31 (Robert Gjerdingen), qui décrit l'émergence de la "Musikpsychologie " (recherche en perception et cognition de la musique) au cours du $\mathrm{XX}^{e}$ siècle, depuis les travaux des structuralistes jusqu'au développement de l'école cognitiviste, en passant par les théories gestaltistes, clôt cette section.

De toute évidence, l'objectif visé par ce livre est de permettre au lecteur de se mettre rapidement au fait des principaux enjeux des différents sous-domaines de la discipline, et non de couvrir un sujet de façon exhaustive. L'ouvrage se veut donc une référence pour les étudiants et professeurs désireux d'acquérir rapidement une vue d'ensemble d'un sujet donné. De fait, on sent chez les auteurs une volonté palpable d'écrire dans une langue rigoureuse et concise, de telle façon que des sujets complexes soient rendus accessibles en une trentaine de pages à des étudiants des cycles supérieurs. Aussi, les auteurs tentent généralement de ratisser large plutôt que de s'enliser dans des points de détail, au risque de devoir se limiter à un condensé qui peut parfois sembler superficiel aux yeux d'un expert. En outre, la grande majorité des chapitres sont rédigés dans un style vivant et narratif, ce qui constitue presque un exploit en soi lorsqu'on considère la relative aridité des thèmes abordés. Certains théoriciens ayant apporté une contribution dans plusieurs domaines sont visités par différents auteurs, chacun choisissant un angle particulier; loin d'être une faiblesse, cette approche met en valeur la diversité des points 
de vue exprimés dans la communauté académique, un aspect d'ailleurs noté par Christensen dans l'introduction (p. 17). À ce titre, l'ouvrage comporte de nombreux liens et références croisées entre chapitres, ce qui rend sa consultation beaucoup plus conviviale, tandis que l'utilisation de tableaux et notes explicatives en médaillon permet d'expliquer des concepts pointus de manière succincte et visuellement attrayante. Notons également le soin apporté à la préparation des illustrations, dont la liste est précisée dans les pages liminaires, et à l'élaboration de chaque index, l'un classé par auteurs, et l'autre par sujets. Ce livre peut donc être consulté de façon ponctuelle, telle une encyclopédie, mais peut également se lire de manière continue, grâce à son écriture dynamique et au fil conducteur qui relie les chapitres entre eux. La théorie musicale s'anime alors sous les yeux du lecteur, comme une discipline aux frontières sans cesse changeantes, un locus de savoir en état de flux constant, au lieu d'apparaître comme un corpus rigide de règles et de méthodes d'analyse. La conception même de ce que représente la théorie musicale dans une culture donnée peut alors être perçue comme un reflet de l'inconscient musical collectif, vers lequel la théorie musicale peut servir, en quelque sorte, de "voie royale " d'accès (p. 21) 3 .

Une des plus grandes qualités du Cambridge History of Western Music Theory est sans contredit sa capacité à placer la théorie musicale, en tant que discipline, à l'intérieur d'un réseau de savoirs plus ou moins connexes tels que les mathématiques (chap. 10), la psychologie (chap. 9 et 31), la linguistique (chap. 29), la philosophie (chap. 3), la pédagogie (chap. 2, 16, 17 et 18), l'astronomie, ou même les sciences occultes (chap. 8). La théorie musicale n'est donc pas considérée de façon isolée, mais comme une discipline se mouvant au sein de profonds courants de pensée qui transcendent les frontières disciplinaires, offrant ainsi au lecteur une riche perspective holistique du savoir, au lieu du point de vue myope souvent encouragé par un curriculum universitaire spécialisé.

On peut peut-être déplorer que le volume, dans l'ensemble, fasse la part belle aux questions reliées à l'harmonie, aux concepts de consonance et de dissonance, et de tonalité, et ne réserve qu'une portion congrue (deux courts chapitres) à l'analyse formelle et motivique. Cet état de choses peut cependant être vu comme le reflet de la perception actuelle de la communauté musicologique en Amérique du Nord, qui, dans l'ensemble, n'a pas accordé aux théories d'analyse formelle et motivique un statut similaire à celui que l'œuvre de Rameau a acquis au plan de l'analyse harmonique, pour ne prendre que cet exemple. De fait, les théories formelles ne sont pas unanimement considérées comme faisant partie intégrante de la discipline, à tel point que Dunsby s'est senti obligé de justifier leur inclusion : "la théorie thématique n'est pas

3 Christensen cite le musicologue allemand Johann Kessel (1766-1823), qui suggérait que l'évolution de la théorie musicale était en elle-même révélatrice de la compréhension changeante des phénomènes musicaux. 
un courant fantaisiste dans la théorie musicale; bien au contraire, il s'agit d'un domaine qui est intimement lié à notre sensibilité artistique » (p. 924) ${ }^{4}$. Il faut cependant applaudir l'ouverture d'esprit de l'éditeur, qui a consacré un chapitre entier (chap. 30) aux théories dites "énergétiques ", dont celles d'Ernst Kurth, un domaine qui a longtemps semblé quelque peu ésotérique aux yeux de certains théoriciens, mais qui a été revigoré depuis quelques années par les travaux de Fred Lerdahl (2001) et Carol Krumhansl (1996), entre autres, qui lui ont donné une dimension plus empirique (p. 949).

La plupart des chapitres réussissent le tour de force d'exposer succinctement les principaux concepts ou enjeux reliés à un sujet particulier, tout en mentionnant les personnalités et ouvrages marquants qui y sont associés, le tout dans un style engageant. Bien évidemment, dans un ouvrage d'une telle envergure, fruit de la collaboration de 32 auteurs, la qualité est parfois inégale d'un chapitre à l'autre, et des différences de style peuvent surgir. Ainsi, le chapitre 12 sur la théorie modale de la Renaissance (Cristle Collins Judd) ne parvient pas tout à fait à intégrer de façon cohérente les nombreux concepts associés à la théorie modale, probablement parce que l'auteure, plutôt que de synthétiser elle-même ces concepts, a choisi de référer le lecteur à d'autres sources; alors que le chapitre 17 (Albert Cohen) paraît quelque peu isolé, comme s'il avait été décidé de laisser une place aux questions liées à l'improvisation et à l'interprétation, mais sans savoir où insérer le chapitre. Par contre, les chapitres 4 (théorie musicale dans l'Antiquité grecque, par Thomas J. Mathiesen), 11 (modes et gammes au Haut Moyen Âge, par David E. Cohen), 21 (théorie rythmique aux XVIII ${ }^{\mathrm{e}}$ et XIX ${ }^{\mathrm{e}}$ siècles, par William E. Caplin), 24 (théorie harmonique de Rameau, par Joel Lester), 25 (théorie harmonique au XIX ${ }^{\mathrm{e}}$ siècle, par David W. Bernstein), et 26 (théorie schenkérienne, par William Drabkin) nous paraissent particulièrement dignes de mention, en ce sens qu'ils procurent au lecteur un sommaire limpide d'un domaine spécifique de la théorie musicale, et leur consultation lui sera assurément d'un grand bénéfice.

Par ailleurs, certains chapitres auraient peut-être dû, si l'on applique à la lettre la classification de Dahlhaus, être placés dans une section différente. Ainsi, le chapitre 31, qui traite de la psychologie de la musique (Robert Gerdingen), aurait tout aussi bien pu être intégré dans les traditions spéculatives, à la suite du chapitre 9, dont il constitue d'ailleurs en quelque sorte le prolongement logique. En outre, de nombreux aspects de l'analyse schenkérienne (chap. 26) semblent relever davantage de l'analyse critique, donc d'une tradition descriptive, que de la tradition dite "régulatrice ". A l'inverse, Burnham nous rappelle, dans le chapitre 28, que les premiers théoriciens de la forme, tels que Koch, Reicha, et A. B. Marx, s'adressaient en fait aux

4 «... thematic theory is not a whimsical trend in music theory, but touches directly on our artistic values ". 
compositeurs et non aux analystes (p. 880), bien que l'analyse formelle soit maintenant solidement ancrée dans la tradition descriptive.

Malgré ces légères réserves concernant la qualité de certaines contributions individuelles et les quelques contorsions, sans doute difficilement évitables, dont souffre l'organisation des différentes sections, le Cambridge History of Western Music Theory constitue une œuvre intellectuelle collective de très haut niveau, et apparaît comme un ouvrage de référence indispensable qui devrait, sans plus tarder, trôner dans la bibliothèque de tout théoricien de la musique qui s'intéresse à l'évolution de la théorie musicale à travers les âges, à ses ramifications idéologiques et socioculturelles, ainsi qu'à ses nombreux liens avec les autres disciplines académiques.

\section{RÉFERENCES}

Christensen, Thomas. 1993. Rameau and Musical Thought in the Enlightenment. Cambridge : Cambridge University Press.

Dahlhaus, Carl. 1968. Untersuchungen über die Entstehung der harmonischen Tonalität. New York: Kassel-Bärenreiter. Traduit en anglais par Robert Gjerdingen sous le titre de Studies on the Origin of Harmonic Tonality (Princeton, NJ : Princeton University Press, 1990).

Fétis, François-Joseph. 1840. Esquisse de l'histoire de l'harmonie, considérée comme art et comme science systématique. Paris : Imprimerie de Bourgogne et Martinet.

Krumhansl, Carol L. 1996. "A Perceptual Analysis of Mozart's Piano Sonata K.282: Segmentation, Tension, and Musical Ideas ». Music Perception 13, $\mathrm{n}^{\circ} 3$ : 401-32.

Lerdahl, Fred. 2001. Tonal Pitch Space. Oxford, NY : Oxford University Press. Riemann, Hugo. 1898. Geschichte der Musiktheorie im IX.-XIX. Jahrhundert. Leipzig : M. Hesse.

Shirlaw, Matthew. 1917. The Theory of Harmony : An Inquiry into the Natural Principles of Harmony, with an Examination of the Chief Systems of Harmony from Rameau to the Present Day. Londres : Novello \& Co.

Zaminer, Frieder et Thomas Ertelt, dir. 1984- . Geschichte der Musiktheorie, 11 vol. (publiés jusqu'à présent, sur un total de 15). Darmstadt : Wissenschaftliche Buchgesellschaft.

BRUNO GINGRAS

Emma Dillon. 2002. Medieval Music-Making and the 'Roman de Fauvel'. New Perspectives in Music History and Criticism. Cambridge: Cambridge University Press. xiv, 304 pp. ISBN 0521813719 (hardcover).

Since the publication in 1990 of a complete facsimile of Paris, Bibliothèque Nationale de France, MS français 146 (hereafter fr.146, to use Dillon's siglum), the most famous witness of the Roman de Fauvel, both text and manuscript, already celebrated in musical and literary circles, has received 